\title{
Research regarding tactile scanning versus optical scanning
}

\author{
Marius Bulgaru, ${ }^{1, *}$, Vlad Bocăneț ${ }^{1}$, and Mircea Muntean $^{1}$ \\ ${ }^{1}$ Technical University of Cluj Napoca, Manufacturing Department, 91944, B Labor, 103-105, Cluj \\ Napoca, Romania
}

\begin{abstract}
In today's fast-moving world, the manufacturing industry must keep up with evolving trends. One such trend that has greatly impacted the manufacturing industry is called Industry 4.0 and is regarded as the fourth industrial revolution. In this revolution one important aspect is that of quality. This paper makes a comparative study between tactile and optical measuring machines in the context of Industry 4.0. As the manufacturing industry must be more flexible and solve problems in a timelier manner, it is important to identify the right technologies appropriate for quality control.
\end{abstract}

\section{The Industry 4.0 concept and quality control}

The concept of Industry 4.0 has been developed since the beginning of the 21 -st century, but the first mention of the concept of Industry 4.0 was made in an article in 2011 published by the German government [1]. It refers to the fourth industrial Revolution, phase through which the manufacture of products and equipment is currently going through.

The emergence and development of this new industrial revolution is due to [2]:

- Increase computing power and storage of computers;

- Development of robots, sensors and simulation programs

- The Internet

- Rapid prototyping;

- Democratization of technology;

- Demand for personalized products;

- Relocation of production in the US and Europe;

- Recovery of production and economic growth

The pursuit of small revolutions in the fields of:

- The application of information and communication technology (ICT) to digitize information and integrate systems to design, develop, manufacture and use of products;

- New software technologies for modeling, simulation, virtualization and digital manufacturing;

- Development of cyber-physical systems to monitor and control physical processes;

- Evolution of 3D and Additive Manufacturing printers to simplify manufacturing;

* Corresponding Author: marius.bulgaru@tcm.utcluj.ro 
- Support for decision making for human operators, the emergence of smart tools and assistance using augmented reality. New forms of human-machine interaction.

The Industry 4.0 concept touches on three dimensions: horizontal integration across the value creation network, end-to-end engineering across the product life cycle and vertical integration and networked manufacturing systems [3]. The digital factory will allow us to optimize all phases of the product lifecycle. The virtual simulations of design and functionality developed in parallel with the planning of manufacturing lead to a much shorter time-to-market, significantly reduce costs and higher quality. Everything will be driven by data analysis. The Digital factory integrates Product Lifecycle Management, Digital Manufacturing, Manufacturing Execution System, and IoT components that communicate feedback from ongoing manufacturing processes or products in operation.

\subsection{D Quality control equipment in Industry 4.0}

Manufacturers of coordinate measuring equipment were not taken by surprise by the new concept. The main aspect of the development of control products can be noted consisting of:

- Development of new scanning equipment and techniques by using computerized tomography;

- Development of optical metrology through new optic and laser sensors;

- Increased measurement accuracy, measurement speeds and the volume of information resulting from control;

- Construction of new control equipment using industrial robots that led to automation of control;

- A strong link between the 3D model and the CNC measurement program via PMIProduct Measuring Information that is part of 3D modeling software;

- Development of soft tools such as PiWeb for evaluating large volumes of control and execution data.

These modifications have integrated the control equipment into the manufacturing lines so today we have functional in line and at line control systems [Fig. 1]

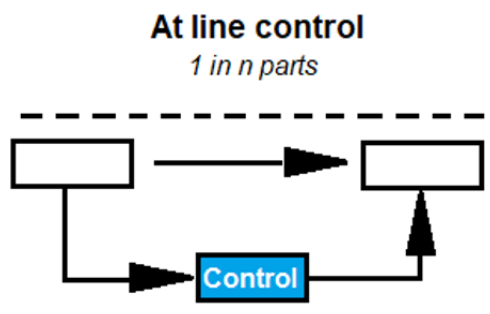

Measuring time: $5-15 \mathrm{~min}$

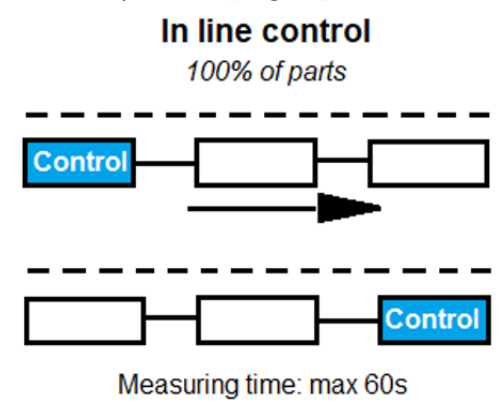

In line control

Fig. 1. At line and in line control

\subsection{The ATOS optical measurement/3D-scanning system}

The ATOS system performs non-contact measurement-scanning operations using the triangulation principle. The options for the at-line control have in composition (Figure 2): the scanning system consisting of two video camera and variable fringe projector and the robot that positions the sensor for the optimal acquisition of the images. The scanned surface results in a point cloud that is processed by triangulation resulting in a mesh. Subsequently, the mesh can be stored in various formats such as IGES or STL. 

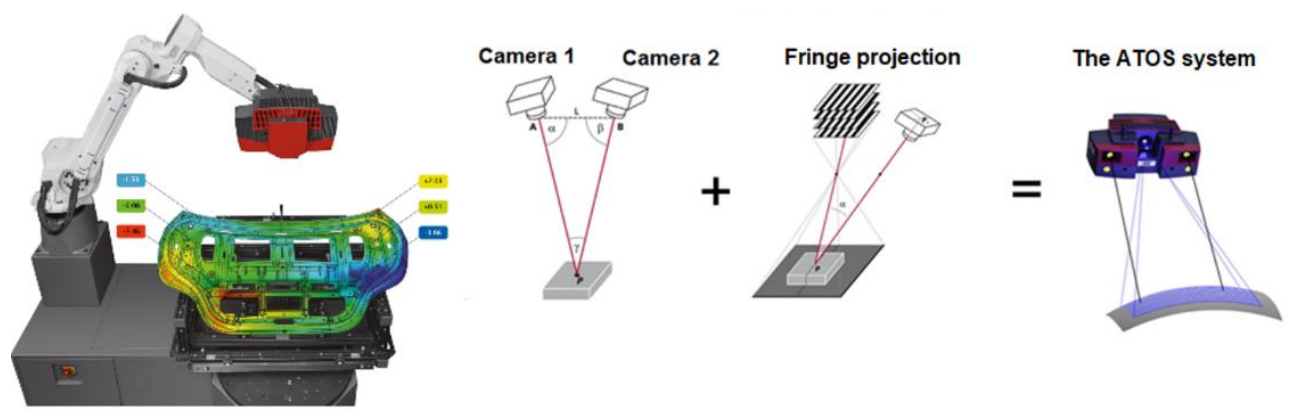

Fig. 2. ATOS System components and operating principle [2]

The GOM Inspect (Geometrical Optical Measurement) software performs the following operations/functions:

- Actual-Nominal comparison

- Alignment

- GD \& T Analysis

- Trend, SPC and deformation analysis

- Reporting

\section{Research on quality control using tactile and optical systems}

\subsection{Other research comparing tactile and optical systems}

There have been few studies done on tactile and optical measuring equipment comparisons. One study [4] compared measured the Halle, KNT4080-30 reference standard artefact by using a tactile probe (a stylus with the tip angle of $90^{\circ}$ and a tip radius of $2 \mu \mathrm{m}$ ), an optical confocal chromatic probe and a laser interferometer. The results of the study show that the tactile measurements are more accurate than the optical ones.

Another study [5] compared a tactile formtester (Mahr Formtester MMQ40) with a white-light interferometer (a Michelson interferometer with a high precision $45^{\circ}$ conical mirror) while measuring profiles of a cylindrical part. Their results show that the point cloud obtained through the two methods are in good agreement.

A third study [6] compared different measurement devices (both tactile and optical) while measuring aspheres. Four aspheres were measured by eight laboratories with tactile , optical point measuring and optical areal measuring machines. The measurement results were compared with a virtual reference topography (VRT). The results show that some measurement systems may have been influenced by systematic errors and that "the data does not suggest that one measurement principle is superior to another" [6]. Another study [7] that found good agreement between measuring machines, compared measurements of microgrears with a micro-CMM, computer tomography, optical CMM and a gear measuring instrument.

\subsection{Research objectives}

The research objective was to conduct a comparative study between the scanning accuracy using tactile systems and optical measurement systems. The necessary requirements for carrying out the scanning operation are evaluated.

The methodology provides the following steps: 
1. Preparation of the gauges. A ring gauge with a diameter of $\varnothing 39.998$ and a plate gauge with a number of $5 \times 5, \varnothing 5.5$ bores were used (Fig. 3.)

2. Scanning the gauges with the two systems.

3. Scanning the plate gauge with the two systems;

4. Processing and comparison of results.

\subsection{Equipment used}

Two high precision measuring machines were used:

1. The Zeiss Prismo Navigator CMM with a VAST Gold measurement sensor

2. The GOM ATOS Scan Box Series 4
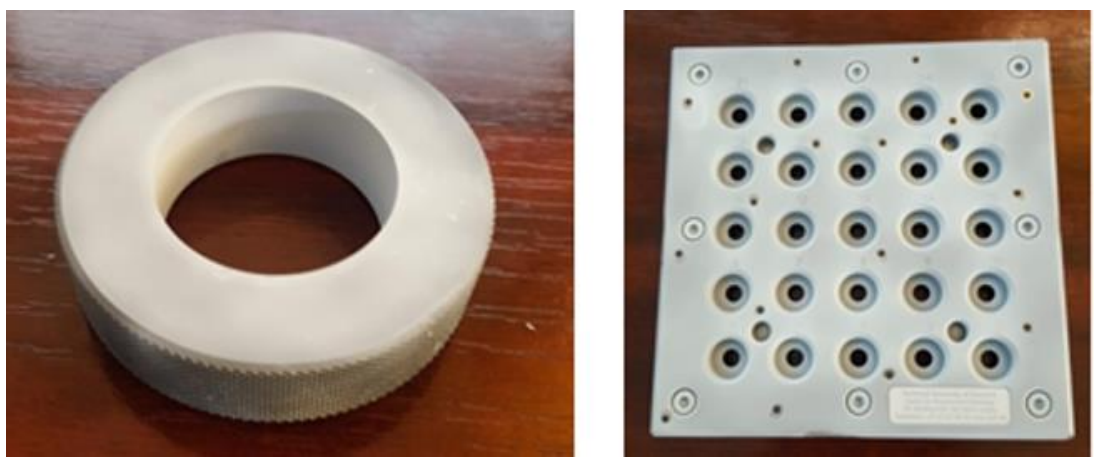

Fig. 3. Ring gauge $\varnothing 39,998$ and the plate gauge with $5 \times 5$, $\varnothing 5$ bores

The preparation of the measurement consisted in degreasing with alcohol, aeration made with an airbrush, covering with a fine powder of mixture of titanium dioxide and isopropylic alcohol for eliminating metal shine, application of markers for proper alignment of scans, calibrating the system, setting the ATOS system to a volume of $80 \mathrm{~mm}^{3}$.

The tactile scanning of the ring calibration was carried out using the CALYPSO program, measuring by scanning three planar sections at the height of 3.0, 9.5 and $16.0 \mathrm{~mm}$. The optical scanning was done with the GOM ATOS v9 [Fig. 4].
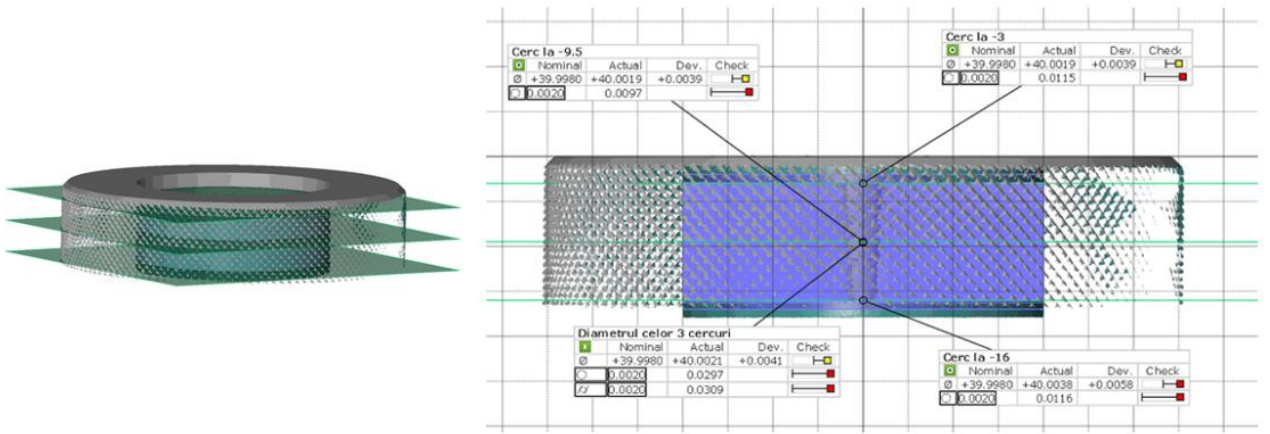

Fig. 4. Measurement of ring gauge Ø 39.998

Tactile scanning of the plate gauge was carried out using the CALYPSO program, measuring by scanning the 25 bores. Optical scanning was achieved with the GOM v.9 program [Fig. 5] 

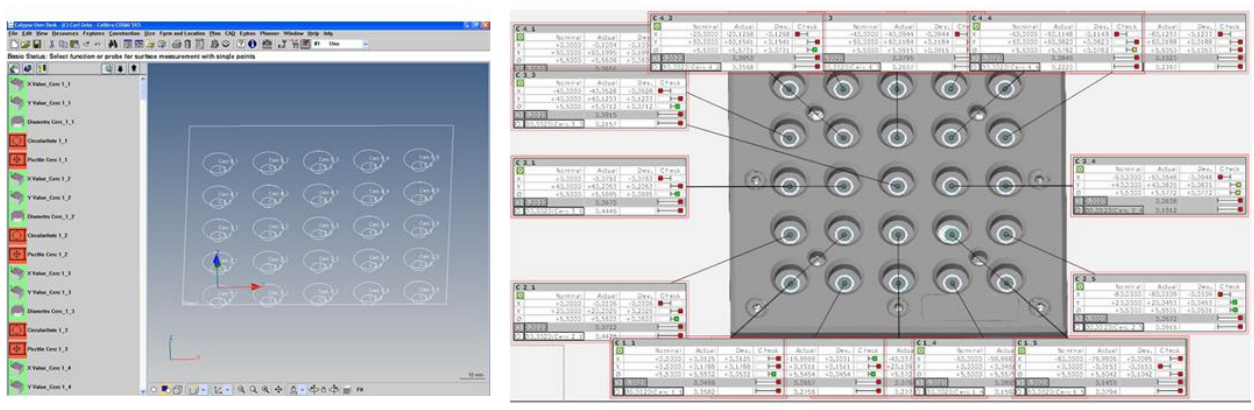

Fig. 5. Plate gauge with $5 \times 5 \varnothing 5.5$ bores

The results obtained when measuring the bore are presented in Table 1 and those obtained by calculating the form deviations (circularity/cylindricity) in Table 2.

Table 1. Comparative study when measuring ring gauge diameters

\begin{tabular}{|c|c|c|c|c|c|c|c|c|}
\hline \multirow{2}{*}{$\begin{array}{c}\text { Ø 39,998 } \\
\text { Gauge }\end{array}$} & $\mathbf{H}=\mathbf{3 . 0}$ & $H=9.5$ & $H=16.0$ & \multirow{2}{*}{$\begin{array}{l}\text { Diameter } \\
\text { Cylinder }\end{array}$} & \multirow{2}{*}{ Min } & \multirow{2}{*}{ Max } & \multirow{2}{*}{ Average } & \multirow{2}{*}{$\begin{array}{c}\Delta \\
\text { St. Dev. }\end{array}$} \\
\hline & & Ø Circle & & & & & & \\
\hline Touch & 40.0002 & 40.0000 & 39.9996 & 39.9997 & 39.9996 & 40.0002 & 39.99987 & 2.75 e- 04 \\
\hline Optical & 40.0019 & 40.0019 & 40.0038 & 40.0021 & 40.0019 & 40.0038 & 40.00242 & $9.22 \mathrm{e}-04$ \\
\hline
\end{tabular}

Table 2. Comparative study of the form deviations of the ring gauge

\begin{tabular}{|c|c|c|c|c|c|c|c|c|}
\hline \multirow{2}{*}{$\begin{array}{c}639,998 \\
\text { Gauge }\end{array}$} & $\mathbf{H}=\mathbf{3 . 0}$ & $\mathbf{H}=9.5$ & $H=16.0$ & \multirow{2}{*}{ Cylindricity } & \multirow{2}{*}{ Min } & \multirow{2}{*}{ Max } & \multirow{2}{*}{ Average } & \multirow{2}{*}{ St. Dev. } \\
\hline & \multicolumn{3}{|c|}{ Circularity } & & & & & \\
\hline Touch & 0.0006 & 0.0005 & 0.0003 & 0.0003 & 0.0006 & 0.0008 & 0.000425 & $1.50 \mathrm{e}-04$ \\
\hline Optical & 0.0115 & 0.0197 & 0.0116 & 0.0115 & 0.0297 & 0.0097 & 0.013575 & $4.08 \mathrm{e}-03$ \\
\hline
\end{tabular}

The analysis of the results shows that tactile measurement is more accurate in terms of nominal value (obtained by calculating arithmetic mean) and in relation to deviations (obtained by calculating Standard deviation and form deviations like circularity/cylindricity). Greater deviations occur in optical scanning due to the fact that when measuring the bores the number of points decreases as the depth of the bore increases.

For the bore plate the following elements were measured: diameters, X, Y position of centers, circularity and position deviation of the bore. Table 7 presents the differences in results.

Table 3. Comparative study of the form deviations of the ring gauge

\begin{tabular}{|c|r|r|r|r|r|r|r|r|r|}
\cline { 3 - 9 } \multicolumn{1}{c|}{} & \multirow{2}{*}{ Nom. } & \multicolumn{2}{|c|}{ Actual average } & \multicolumn{2}{c|}{ Actual Max. } & \multicolumn{2}{c|}{ Actual Min } & \multicolumn{2}{c|}{ St. Dev. } \\
\cline { 3 - 10 } \multicolumn{1}{c|}{} & & ATOS & \multicolumn{1}{c|}{ CMM } & \multicolumn{1}{c|}{ ATOS } & \multicolumn{1}{c|}{ CMM } & \multicolumn{1}{c|}{ ATOS } & CMM & \multicolumn{1}{c|}{ ATOS } & CMM \\
\hline Diameter & 5,500 & 5.5693 & 5.5238 & 5.6050 & 5.5260 & 5.5405 & 5.5217 & 0.0165 & 0.0014 \\
\hline X position & 0.000 & & & 0.1713 & 0.1509 & -0.1426 & -0.0181 & 0.0828 & 0.0596 \\
\hline Y position & 0.000 & & & 0.2248 & 0.2018 & -0.0153 & 0.0356 & 0.0643 & 0.0586 \\
\hline Circularity & 0.002 & 0.0777 & 0.0427 & 0.1455 & 0.0471 & 0.0222 & 0.0395 & 0.0240 & 0.0020 \\
\hline Position & 0200 & 0.2931 & 0.2815 & 0.4958 & 0.4625 & 0.0794 & 0.0785 & 0.1152 & 0.1145 \\
\hline
\end{tabular}

It is noted that in the tactile measurement the results obtained have a lower measurement uncertainty. The average value, circularity and extremes (maximum and minimum values) are closer together. Also, the standard deviation is greatly diminished.

The same trend is also found in the case of position deviations, but differences are not very high between the two sensors.

Similar results were found in the case of the plate gauge (Tables 4). 
Table 4. Results for the plate gauge

\begin{tabular}{|c|c|c|c|c|c|c|}
\hline Gauge & \multicolumn{3}{|c|}{ Diam. 5.5 mm } & \multicolumn{3}{c|}{ Diam. 12 mm } \\
\hline & ATOS & MMC & Nominal & ATOS & MMC & Nominal \\
\hline Average diameter & 5.5695 & 5.5238 & 5.5 & 12.0897 & 12.0929 & 12 \\
\hline St. Dev. Diameter & 0.0165 & 0.0014 & & 0.0044 & 0.0042 & \\
\hline Circularity & 0.0770 & 0.0429 & 0 & 0.0195 & 0.0500 & 0 \\
\hline St. Dev. Circularity & 0.0240 & 0.0020 & & 0.0057 & 0.0061 & \\
\hline
\end{tabular}

\section{Conclusions}

The current study found that tactile measurement leads to more precise results in terms of both dimensions and variance when compared to optical methods.

The experimental research between the two scanning methods also outlined the significant differences between tactile and optical measurements. The tactile method has the advantages of being a recognised technology, it is fast for a low number of points and portal CMMs have a high accuracy. At the same time, it has the disadvantage of taking a long time when scanning surfaces, requires clamping devices, it requires the alignment of the part in the machine coordinate system and cannot be used for any materials (e.g. elastic or soft materials).

On the other hand, the optical methods allow the fast scanning of entire surfaces and automation of the control operation. Furthermore, the system is mobile and can be used in a production environment, it has a large measuring volume and allows the use of multiple alignment systems. It also has the disadvantage of requiring preparatory operations such as covering shiny, transparent or black parts, having a thermally instable housing and presenting measurement deviations when the optical axis is not perpendicular to the surface.

\section{References}

2 A.C. Pereira, F. Romero, A review of the meanings and the implications of the Industry 4.0 concept, Procedia Manufacturing, 13, 1206-1214, (2017)

3 DIGITAL TWIN E-book digitization production switching to Industry 4.0 (2019)

4 T. Stock , \& G.Seliger,. Opportunities of sustainable manufacturing in industry 4.0. Procedia CIRP, 40, 536-541, (2016)

5 H. Nouira, J. A. Salgado, N. El-Hayek, S. Ducourtieux, A. Delvallée, and N. Answer, Setup of a high-precision profilometer and comparison of tactile and optical measurements of standards, Measurement Science and Technology 25, no. 4: 044016, (2014) .

6 M. R. Viotti, A. Albertazzi, A. V. Fantin, and A. Dal Pont, Comparison between a white-light interferometer and a tactile formtester for the measurement of long inner cylindrical surfaces, Optics and Lasers in Engineering 46, no. 5 396-403, (2008)

7 R. Schachtschneider, I. Fortmeier, M. Stavridis, J. Asfour, G. Berger, R. B. Bergmann, A. Beutler et al. Interlaboratory comparison measurements of aspheres, Measurement Science and Technology 29, no. 5, 055010, (2018).

8 S. Jantzen, M. Stein, A. Dietzel, K. Kniel, Microgear measurement standards: Comparing tactile, optical and computed tomography measurements, American Gear Manufacturers Association Fall Technical Meeting (2018) 